\title{
REVISTA DE ADMINISTRACIÓN PÚBLICA
}

\author{
Director: Eduardo GARCfA DE ENTERRfA \\ Secretaria: Carmen CHINCHILla Marin \\ SUMARIO DEL NÚM. 139 (enero-abril 1996)
}

\section{ESTUDIOS}

J. L. MEILÁN GiL: El dominio natural y la legislación de costas.

F. LOPEz Ramon: Consideraciones jurídicas sobre la función de las Comunidades Autónomas en la ordenación ferroviaria.

A. SÁnCHEZ BlanCo: Convergencia interadministrativa en la acción pública sobre el territorio. A propósito del dictamen de la Comisión de expertos sobre urbanismo y el Plan director de infraestructuras 1993-2007.

J. GaRCta MORILlo: La versatilidad de lo básico.

\section{JURISPRUDENCIA}

I. Comentarios monográficos

M. BEATo EsPEjo: El medio ambiente urbano y la convivencia ciudadana: El ruido por el consumo de bebida en la vía príblica.

M. SuÁREz OJEDA: Sobre la cesión tácita de las obras de urbanización en la jurisprudencia del Tribunal Supremo.

J. M. Rodriguez de SANTIAGo: Otro embate contra el carácter revisor: el recurso contencioso-administrativo ante el incumplimiento por el Jurado Provincial de Expropiación de la obligación de fijar el justiprecio en plazo. STC 136/1995, de 25 de septiembre.

II. Notas

Contencioso-administrativo

A) En general (T. FONT LLOVET y J. TORNOS MAS).

B) Personal (R. ENTRENA CUESTA).

CRÓNICA ADMINISTRATIVA

DOCUMENTOS Y DICTÁMENES

BIBLIOGRAFIA

\section{PRECIOS DE SUSCRIPCIÓN ANUAL}

España 5.800 ptas.

Extranjero

$61 \$$

Número suelto España

2.100 ptas.

Nümero suelto Extranjero

$22 \$$

Suscripciones y números sueltos:

CENTRO DE ESTUDIOS CONSTITUCIONALES

Fuencarral, 45, 6."

28004 MADRID 


\title{
REVISTA DE INSTITUCIONES EUROPEAS
}

\author{
Directores: Manuel Diez de Velasco, Gil Carlos Rodriguez Iglesias \\ y Araceli Mangas Martin \\ Directora ejecutiva: Araceli MANGAS MARTIN \\ Secretaria: Nila TORRES UGENA
}

SUMARIO DEL VOLUMEN 23, NÚM. 1 (enero-abril 1996)

\section{ESTUDIOS}

Pierre PESCATORE: La interpretación del Derecho Comunitario por el juez nacional.

Iñaki LASAGABASTER HeRrarte: Ciudadanía y Tratado de Schegen: naturaleza jurídica de las disposiciones del Comité Ejecutivo.

Andreas HILDENBRAND SCHEI: Nuevas iniciativas de la Unión Europea en materia de ordenación del territorio.

\section{NOTAS}

Xavier Pons Rafols: Participación de la Comunidad Europea en la Comisión de Desarrollo sostenible de las Naciones Unidas.

fñigo Bullain: Europa y la Conferencia Intergubernamental de 1996.

Jaime SANCHEZ SANTIAGO: Difamar en Europa las implicaciones del asunto Sbevill.

Fernando GonzAlez Botija: La tutela cautelar en el marco de la Política Común de Pesca.

\section{JURISPRUDENCIA}

Tribunal de Justicia de las Comunidades Europeas.

CRÓNICA

BIBLIOGRAFÍA

DOCUMENTACION

PRECIOS DE SUSCRIPCIÓN ANUAL

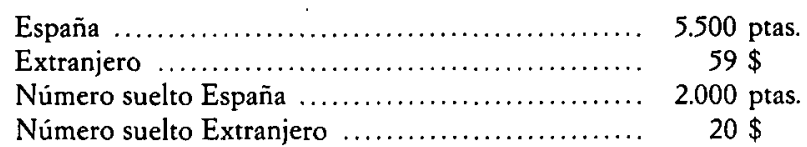

Suscripciones y números sueltos:

CENTRO DE ESTUDIOS CONSTITUCIONALES

Fuencarral, 45, 6.

28004 MADRID 


\title{
REVISTA DE ESTUDIOS POLÍTICOS
}

\section{(NUEVA ÉPOCA)}

\author{
DIRECTOR: Pedro DE Vega GaRCIA \\ SECRETARIO: Juan J. SOLOZÁBAL ECHAVARRIA
}

SUMARIO DEL NÚM. 91 (enero-marzo 1996)

\section{ESTUDIOS}

Javier CoRCuera AtIENZA: Estado y economia en época de crisis: las privatizaciones.

Javier RoIz: Hobbes como coartada del pensamiento borbónico.

José Félix Tezanos: Comunidad y sociedad como paradigmas politicos.

Gerardo Ruiz-Rico Ruiz: Los derechos de la minorias religiosas, lingüisticas y técnicas en el ordenamiento constitucional español.

José Antonio LOPEZ GARCIA: La presencia de Carl Scbmitt en España.

\section{NOTAS}

Adela Mesa: Los cargos de designación política ante el proceso de cambio en la Administración autonómica vasca.

Azucena Rodrfguez Ávarez: Aproximación a la idea de urepública» en la Francia revolucionaria.

Hugo D. BerTtN y Juan Carlos CoRBETTA: La eficacia y efectividad de la política de seguridad interna del gobierno justicialista: 1975.

Caterina GaRCia SEgURA: La actividad exterior de las entidades políticas subestatales.

Quim BRUGUE y Ricard GomA: El impacto de los partidos sobre las políticas sociales. Una perspectiva comparada.

\section{CRONICAS Y DOCUMENTACION}

Geoffrey K. Roberts: Sistema de partidos y Parlamento en Gran Bretaña: 1994.

Ismael CRESPO y Pablo Mieres: Las elecciones uruguayas de 1994: Continuidad en la transformación del sistema partidista.

\section{RECENSIONES. NOTICIAS DE LIBROS}

\section{PRECIOS DE SUSCRIPCIÓN ANUAL}

España

Extranjero

Número suelto España

Número suelto Extranjero
5.850 ptas.

$61 \$$

1.600 ptas.

$22 \$$

Suscripciones y números sueltos:

\section{CENTRO DE ESTUDIOS CONSTITUCIONALES}

Fuencarral, 45, 6."

28004 MADRID 


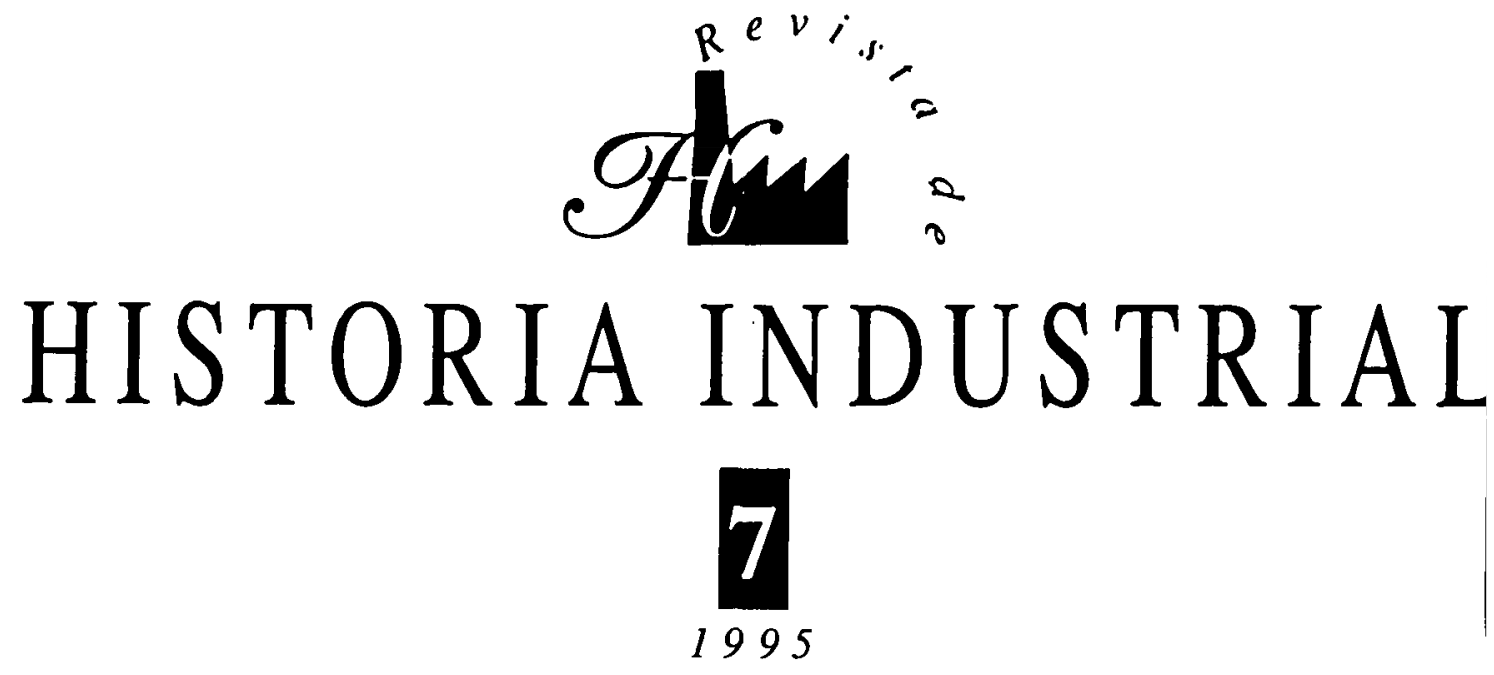

A. Sánchez Picón, Modelos tecnológicos en
- de 1906. I. Bartolomé, Los lími- tes de la hulla blanca en vísperas - de la Guerra Civil. E. SAN Román, - El nacimiento de la SEAT: autarcidaden España. M. SABAtéSort, La - quía e intervención del INI. DIÁlogoS Y ENTREVISTAS: LouIS BERGERON, Arqueología Industrial, pasado y presente. Por G. DOREL - FerRé. • NOTAS DE INVESTIGACIÓN • RECENSIONES 


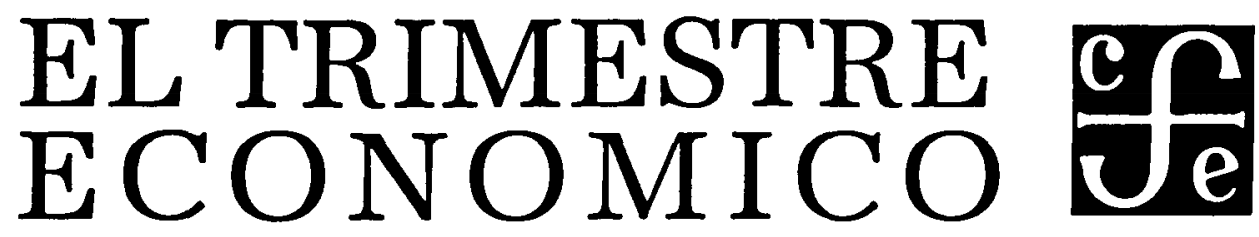

COMITÉ DICTAMINADOR: Alejandro Castañeda, Pablo Cotler, Raùl Garcla, Raúl Livas, Luda Segovia (Secretaria Técnica), Rodolfo de la Torre. CONSEJO EDITORIAL: Edmar L. Bacha, José Blanco, Gerardo Bueno, Enrique Cárdenas, Arturo Fernández, Ricardo Ffrench-Davis, Enrique Florescano, Roberto Frenkel, Ricardo Hausmann, Alejandro Hernández, Albert O. Hirschman, David Ibarra, Felipe Larrain, Francisco Lopes, Guillermo Maldonado, Rodolfo Manuelli, José A. Ocampo, Joseph Ramos, Luis Angel Rojo Duque, Gert Rosenthal, Francisco Sagasti, Jaime José Serra, Jesús Silva Herzog Flores, Osvaldo Sunkel, Carlos Tello, Sweder van Winjberger.

Director: Rodolfo de la Torre. Subdirector: Raúl Livas Secretario de Redacción: Guillermo Escalante A.

Vol. LXII (4)

México, Octubre-Diciembre de 1995

Núm. 248

ARTICULOS

Luls E. Arjona Béjar

Manuel R. Agosin

Gonzalo Castañeda Ramos

NOTAS Y COMENTARIOS: Joseph Hodara, Prebisch: Diez años despues. DOCUMENTOS: Estudio económico de la OCDE, México

EL TRImestre EConómico aparece en los meses de enero, abril, julio y octubre. La suscripción en México cuesta $\$ 100.00$. Número suelto $\$ 35.00$. Disquetes con el índice general (por autores y lemático) de los números 1-244, $\$ 26.00$ (4.49 dls.).

\section{Precio de suscripción por un año, 1996}

$$
\begin{gathered}
\text { España, Centro y Sudamérica } \\
\text { (dólares) }
\end{gathered}
$$

Resto del mundo
(dolares)

\section{Personal}

Número suelto

Universidades, bibliotecas e instituciones Número suelto
35.00

12.00

42.00

18.00

42.00

30.00

Fondo de Cultura Económica, carretera Picacho Ajusco 227, Col. Bosques del Pedregal, 14200 México, D.F. Suscripciones y anuncios: teléfono 2274670 , señora Irma Barrón. 


\section{NOTICIARIO DE HISTORIA $A G R A R I A$}

\section{0}

\section{Estudios}

CORONA, G.

La lucha por el individualismo agrario en el Mezzogıorno italiano a finales del siglo XVIII

$$
\text { ERDOZÁIN AZPILICUETA, P. }
$$

Familia. patrimonio y procesos de trabajo en la Navarra Media

Occidental a finales del siglo $X I X$

GaRRABOU, R.; PASCUAL, P.: PUJOL, J. y SAGUeR, E.

Polencialidad producliva y rendimientos cerealicolas en la agricultura calalana contemporánea (1820-1935)

\section{GEA (Grupo de Estudios Agrarios)}

Transformaciones agrarias y cambios en la funcionalidad de los poderes locales en la Alla Andalucia, 1750-1950

\section{LANA BERASAIN, J.M.}

Trabajo, técnica y mercado en la viticultura navarra: los viñedos del Marqués de San Adrián en Monteagudo durante el sigio XIX

PAN-MONTOJO, J.

La administración agraria en Espana. 1847-1907

\section{Crítica y reseña de libros - Bibliografía}

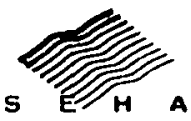

SEMINARIO

DE HISTORIA AGRARIA
Suscripciones:

SEMINARIO DE HISTORIA AGRARIA (SEHYA)

Facultad de Ciencias Económicas y Empresariales

Universidad de Zaragoza

Cl Dr. Cerrada, 1. E.50005 Zarago:za

Telelonos (976) 761841 o 761787

Nümero Fax (976) 761770

E-mail: germariomecon unizar.es

\section{Intercambia:}

Secrotariado de Publicaciones o

Inlercambio Cientilico

UNIVERSIDAD DE MURCIA

Cl. Santo Cristo, 1

30001 MURCIA (ESPAÑA) 


\section{ALIANZA} EDITORIAL

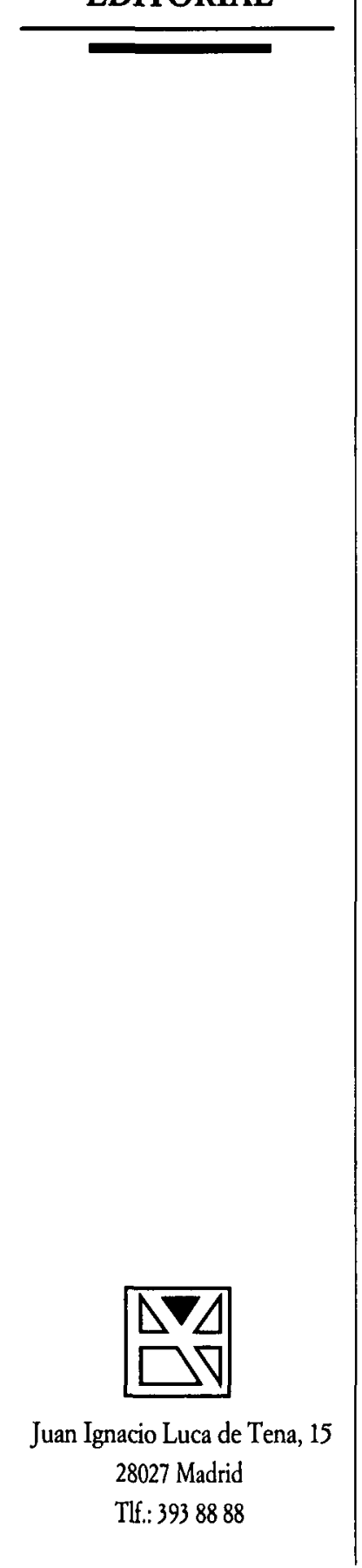

\section{ECONOMÍA}

Ramón Tamames y

Santiago Gallego

DICCIONARIO DE

ECONOMf́ Y FINANZAS

LS 222

\section{Anne O. Krueger}

LA ECONOMfA POLfTICA DE LA REFORMA EN LOS PAÍSES EN

DESARROLLO AE 20

Richard Layard, Stephen Nickell y Richard Jackman LA CRISIS DEL PARO AE 21

Jordi Canals BANCOS UNIVERSALES Y DIVERSIFICACIÓN EMPRESARIAL $A E 22$

\section{SOCIALES}

David Lyon

EL OJO ELECTRÓNICO $\mathrm{El}$ auge de la sociedad de la vigilancia TES 4

\section{Robert Wuthnow}

ACTOS DE COMPASIÓN Cuidar de los demás y ayudarse a uno mismo

LS 195
Víctor Pérez-Díaz

ESPAÑA PUESTA A PRUEBA 1976-1996 AAct. 2

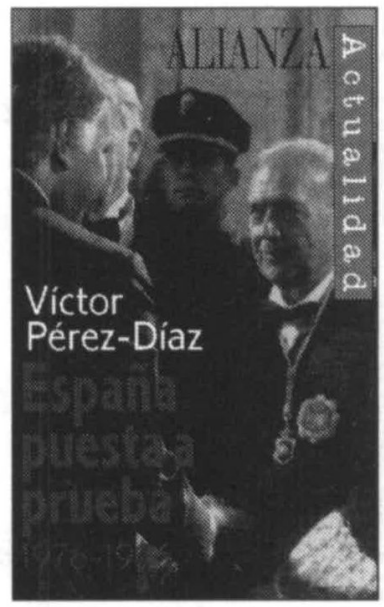

\section{HISTORIA}

Eduard Fuchs

HISTORIA ILUSTRADA

DE LA MORAL SEXUAL

1. Renacimiento

2. Época galante

3. Época burguesa

LS 218-219-220

Concepción de Castro

CAMPOMANES

Estado y reformismo ilustrado AU 838

\section{David R. Ringrose}

ESPAÑA, 1700-1900:

EL MITO DEL FRACASO $A U 850$ 


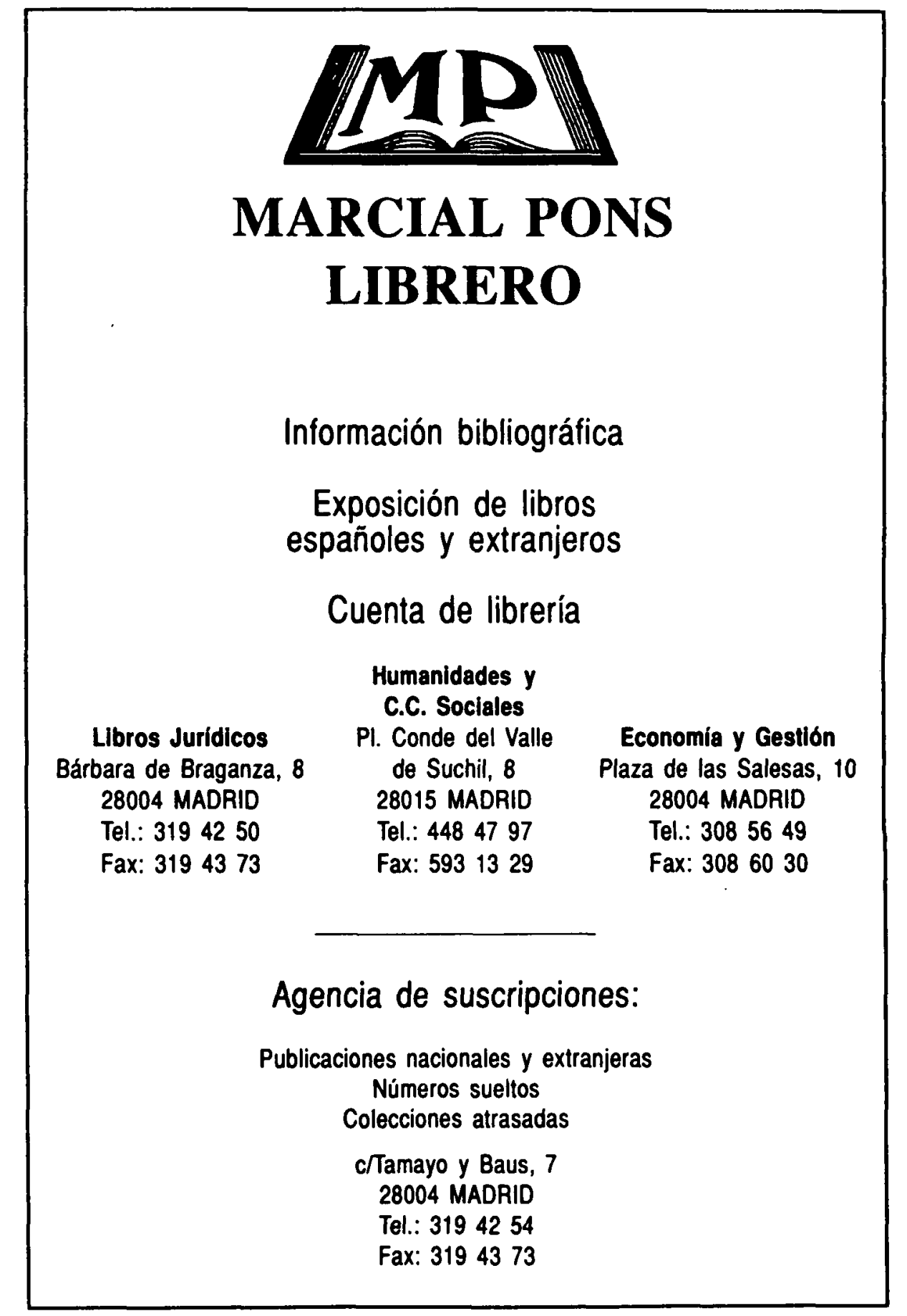




\section{PATRONATO}

Gabriel Tortella (Presidente)

Luis Aguiar Luque (Director del Centro de Estudios Constitucionales)

Rafael Martinez Alés (Director de Alianza Editorial)

Leandro Prados de la Escosura (Universidad Carlos III)

Felipe Ruiz Martin (Presidente Honorario de la Asociación de Historia Económica)

Julio Segura Sánchez (Director de la Fundación Empresa Pública) 


\section{Revista de

